\title{
Non-local electron transport and cross-resistance peak in NSN heterostructures
}

\author{
Mikhail S. Kalenkov \\ I.E. Tamm Department of Theoretical Physics, P.N. Lebedev Physics Institute, 119991 Moscow, Russia \\ Andrei D. Zaikin \\ Forschungszentrum Karlsruhe, Institut für Nanotechnologie, 76021, Karlsruhe, Germany and \\ I.E. Tamm Department of Theoretical Physics, P.N. Lebedev Physics Institute, 119991 Moscow, Russia
}

\begin{abstract}
We develop a microscopic theory describing the peak in the temperature dependence of the nonlocal resistance of three-terminal NSN devices. This peak emerges at sufficiently high temperatures as a result of a competition between quasiparticle/charge imbalance and subgap (Andreev) contributions to the conductance matrix. Both the height and the shape of this peak demonstrate the power law dependence on the superconductor thickness $L$ in contrast to the zero-temperature non-local resistance which decays (roughly) exponentially with increasing $L$. A similar behavior was observed in recent experiments.
\end{abstract}

Non-local effects in coherent electron transport across hybrid structures composed of a superconductor (S) attached to two normal terminals $(\mathrm{N})$ have recently become a subject of intensive experimental $1,2,3$ and theoretical $4,5,6,7,8,9,10,11,12$ investigations. Provided the distance $L$ between two N-terminals (see Fig. 1) is smaller than (or comparable with) the superconducting coherence length $\xi$, two non-local processes contribute to electron transport through such NSN devices. One process corresponds to direct electron transfer (DET) between two N-metals through a superconductor. Another process is the so-called crossed Andreev reflection (CAR): An electron penetrating into the superconductor from the first N-terminal forms a Cooper pair together with another electron from the second N-terminal in which case a hole goes into the second N-metal. A non-trivial interplay between DET and CAR yields a rich variety of features observed in recent experiments ${ }^{1,2,3}$.

Here we focus our attention only on one of such features, a pronounced peak in the temperature dependence of the non-local resistance observed in three-terminal NSN structures 1.3 and attributed to charge imbalance effects. Very recently Golubev and one of the authors ${ }^{11}$ offered a theory for this phenomenon interpreting the nonlocal resistance peak as a result of a competition between the contributions of charge imbalance and Andreev reflection. A striking experimental observation ${ }^{3}$ is that the height of the resistance peak depends on the distance $L$ between N-terminals much weaker than the corresponding low temperature cross-resistance which was found to decay (approximately) exponentially $1.3 \propto \exp (-L / \xi)$ in agreement with theoretical predictions 4,5,6,8,12. Note that due to the restriction $L \lesssim \xi$ it was not possible to address the length dependence of the resistance peak ${ }^{1,3}$ within the model ${ }^{11}$.

Below we will employ the model of three-terminal NSN structures with ballistic electrodes ${ }^{8}$ which allows for a complete non-perturbative solution of the problem for all values of $L$. We will specifically address the temperature dependence of the non-local resistance $R_{12}(T)$ and demonstrate that the height of the charge imbalance peak

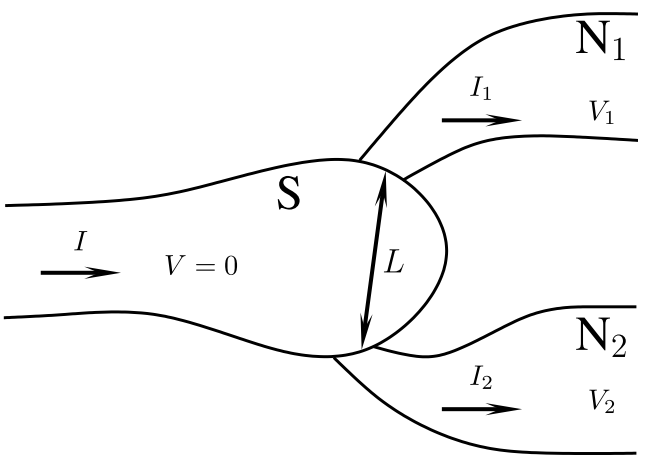

FIG. 1: Schematics of our NSN device.

scales with $L$ exactly as the corresponding normal state resistance $R_{N_{12}}$. We believe that this observation might help to account for recent experimental findings ${ }^{3}$. In addition we will argue that - within the model studied here - the charge imbalance peak for $R_{12}(T)$ can occur only in the case of weakly transmitting NS interfaces and it quickly disappears as the interface transmissions increase beyond the tunneling limit.

The NSN structure under consideration is depicted in Fig. 1. We will assume that all electrodes are ballistic and that both NS interfaces (with cross-sections $\mathcal{A}_{1}$ and $\mathcal{A}_{2}$ ) have arbitrary transmissions $D_{1}$ and $D_{2}$ ranging from zero to one. The distance between these interfaces $L$ as well as other geometric parameters are assumed to be much larger than $\sqrt{\mathcal{A}_{1,2}}$, i.e. effectively both contacts are metallic constrictions. At the same time the number of conducting channels $\mathcal{N}_{1,2}=p_{F}^{2} \mathcal{A}_{1,2} / 4 \pi$ in each contact is assumed to be large.

For convenience, we will set the electric potential of the S-electrode equal to zero, $V=0$. In the presence of bias voltages $V_{1}$ and $V_{2}$ applied to two normal electrodes (see Fig. 1) the currents $I_{1}$ and $I_{2}$ will flow through $\mathrm{SN}_{1}$ and $\mathrm{SN}_{2}$ interfaces. These currents can be evaluated with the aid of the quasiclassical formalism of nonequilibrium Green-Eilenberger-Keldysh functions $13 \hat{g}^{R, A, K}$. For ballistic electrodes considered here the Eilenberger equa- 
tions take the form

$$
\begin{aligned}
{\left[\varepsilon \hat{\tau}_{3}+e V(\boldsymbol{r}, t)-\hat{\Delta}(\boldsymbol{r}, t), \hat{g}^{R, A, K}\left(\boldsymbol{p}_{F}, \varepsilon, \boldsymbol{r}, t\right)\right]+} \\
+i \boldsymbol{v}_{F} \nabla \hat{g}^{R, A, K}\left(\boldsymbol{p}_{F}, \varepsilon, \boldsymbol{r}, t\right)=0,
\end{aligned}
$$

where $[\hat{a}, \hat{b}]=\hat{a} \hat{b}-\hat{b} \hat{a}, \varepsilon$ is the quasiparticle energy, $\boldsymbol{p}_{F}=$ $m \boldsymbol{v}_{F}$ is the electron Fermi momentum vector and $\hat{\tau}_{3}$ is the Pauli matrix. The functions $\hat{g}^{R, A, K}$ also obey the normalization conditions $\left(\hat{g}^{R}\right)^{2}=\left(\hat{g}^{A}\right)^{2}=1$ and $\hat{g}^{R} \hat{g}^{K}+$ $\hat{g}^{K} \hat{g}^{A}=0$. Here and below the product of matrices is defined as time convolution.

The matrices $\hat{g}^{R, A, K}$ and $\hat{\Delta}$ are $2 \times 2$ matrices in the Nambu space

$$
\hat{g}^{R, A, K}=\left(\begin{array}{ll}
g^{R, A, K} & f^{R, A, K} \\
\tilde{f} R, A, K & \tilde{g}^{R, A, K}
\end{array}\right), \quad \hat{\Delta}=\left(\begin{array}{cc}
0 & \Delta \\
-\Delta^{*} & 0
\end{array}\right),
$$

and $\Delta$ is the BCS order parameter. Without loss of generality below we choose $\Delta$ to be real. The current density is related to the Keldysh function $\hat{g}^{K}$ by the standard formula

$$
\boldsymbol{j}(\boldsymbol{r}, t)=-\frac{e N_{0}}{4} \int d \varepsilon\left\langle\boldsymbol{v}_{F} \operatorname{Sp}\left[\hat{\tau}_{3} \hat{g}^{K}\left(\boldsymbol{p}_{F}, \varepsilon, \boldsymbol{r}, t\right)\right]\right\rangle,
$$

where $N_{0}=m p_{F} / 2 \pi^{2}$ is the density of state at the Fermi level and angular brackets $\langle\ldots\rangle$ denote averaging over the Fermi momentum.

The above equations should be supplemented by the boundary conditions describing electron scattering at NS interfaces. Assuming specular reflection at both $\mathrm{SN}_{1}$ and $\mathrm{SN}_{2}$ interfaces we introduce their transmission probabilities $D_{1,2}\left(p_{x_{1,2}}\right) \equiv 1-R_{1,2}\left(p_{x_{1,2}}\right)$ (where $p_{x_{1}}\left(p_{x_{2}}\right)$ is the component of $\boldsymbol{p}_{F}$ normal to $\mathrm{SN}_{1}\left(\mathrm{SN}_{2}\right)$ interface) and employ the standard Zaitsev boundary conditions 14 in order to match quasiclassical Green functions at both sides of each of the two interfaces. Deep inside metallic electrodes $\mathrm{S}, \mathrm{N}_{1}$ and $\mathrm{N}_{2}$ the Green functions should approach their equilibrium values $\hat{g}^{R, A}= \pm\left(\varepsilon \hat{\tau}_{3}-\hat{\Delta}\right) / \Omega^{R, A}$ in a superconductor and $\hat{g}^{R, A}= \pm \hat{\tau}_{3}$ in normal metals, $\Omega^{R, A}=$ $\sqrt{(\varepsilon \pm i \delta)^{2}-\Delta^{2}}$. For the Keldysh functions far from interfaces we have $\hat{g}^{K}=\tanh \left[\left(\varepsilon+e V \hat{\tau}_{3}\right) / 2 T\right]\left(\hat{g}^{R}-\hat{g}^{A}\right)$, where $V=0, V_{1}$ and $V_{2}$ respectively in $\mathrm{S}, \mathrm{N}_{1}$ and $\mathrm{N}_{2}$ electrodes.

The general solution of the problem within the above formalism was described in details in Ref $\underline{\underline{8}}$. Here we only point out that the accuracy of the above formalism in the case of double-barrier structures under consideration is justified simultaneously by the two conditions ${ }^{8,15}$ : $\mathcal{A}_{1,2} \ll L^{2}$ and $\mathcal{N}_{1,2} \gg 1$.

At low voltages $e V_{1,2} \ll T_{c}$ we obtain

$$
\begin{gathered}
I_{1}=G_{11}(T) V_{1}-G_{12}(T) V_{2}, \\
I_{2}=-G_{21}(T) V_{1}+G_{22}(T) V_{2} .
\end{gathered}
$$

where $G_{12}(T)$ and $G_{21}(T)$ are the non-local conductances of our NSN device ${ }^{8}$ :

$$
\begin{aligned}
& G_{12}(T)=G_{21}(T)=\frac{G_{N_{12}}}{4 T} \int \frac{d \varepsilon}{\cosh ^{2}(\varepsilon / 2 T)} \\
& \quad \times\left(1-\mathcal{R}_{1}|a|^{2}\right)\left(1-\mathcal{R}_{2}|a|^{2}\right) \frac{1-\tanh ^{2} i L \Omega / v_{F}}{P\left(\mathcal{R}_{1}, \mathcal{R}_{2}\right)} .
\end{aligned}
$$

Here we defined $\mathcal{D}_{1,2} \equiv 1-\mathcal{R}_{1,2}=D_{1,2}\left(p_{F} \gamma_{1,2}\right)$ and $p_{F} \gamma_{1(2)}$ is normal to the first (second) interface component of the Fermi momentum for electrons propagating straight between the interfaces, $\Omega=\sqrt{\varepsilon^{2}-\Delta^{2}}$, $P\left(R_{1}, R_{2}\right)=\mid 1-R_{1} R_{2} a^{2}-Q\left[\varepsilon\left(1+R_{1} R_{2} a^{2}\right)+\Delta a\left(R_{1}+\right.\right.$ $\left.\left.R_{2}\right)\right]\left.\right|^{2}, Q=\Omega^{-1} \tanh i L \Omega / v_{F}, a=(\Omega-\varepsilon) / \Delta$,

$$
G_{N_{12}}=\frac{8 \gamma_{1} \gamma_{2} \mathcal{N}_{1} \mathcal{N}_{2} \mathcal{D}_{1} \mathcal{D}_{2}}{R_{q} p_{F}^{2} L^{2}}
$$

is the non-local conductance in the normal state, $R_{q}=$ $2 \pi / e^{2}$ is the quantum resistance unit.

The conductance $G_{11}(T)$ of the first interface is dominated by the standard BTK expression ${ }^{16}$

$G_{11}(T)=\frac{\mathcal{N}_{1}}{R_{q} T} \int \frac{\left(1+|a|^{2}\right) d \varepsilon}{\cosh ^{2}(\varepsilon / 2 T)}\left\langle\frac{\left|v_{x_{1}}\right|}{v_{F}} D_{1} \frac{1-R_{1}|a|^{2}}{\left|1-R_{1} a^{2}\right|^{2}}\right\rangle$,

while a non-local correction to (8) is small in the parameter $\mathcal{A}_{2} / L^{2}$ and will be omitted here. The conductance $G_{22}(T)$ of the second interface is defined analogously.

In the temperature interval $e^{-\Delta / T} \ll 1$ we obtain following expressions for the conductances

$$
\begin{array}{r}
G_{11}(T)=G_{11}(0)+G_{N_{11}} \sqrt{2 \pi} \sqrt{\frac{\Delta}{T}} e^{-\Delta / T}, \\
G_{12}(T)= \begin{cases}2 G_{N_{12}} e^{-\Delta / T}, & \mathcal{D}_{1} \mathcal{D}_{2} \ll e^{-\Delta / T}, \\
G_{12}(0), & T \ll \frac{\Delta}{\ln \left(1 /\left[\mathcal{D}_{1} \mathcal{D}_{2}\right]\right)},\end{cases}
\end{array}
$$

where

$$
\frac{G_{12}(0)}{G_{N_{12}}}=\frac{\mathcal{D}_{1} \mathcal{D}_{2}\left(1-\tanh ^{2} L \Delta / v_{F}\right)}{\left[1+\mathcal{R}_{1} \mathcal{R}_{2}+\left(\mathcal{R}_{1}+\mathcal{R}_{2}\right) \tanh L \Delta / v_{F}\right]^{2}},
$$

$G_{N_{11}}=\frac{2 \mathcal{N}_{1}}{R_{q}}\left\langle\frac{\left|v_{x_{1}}\right|}{v_{F}} D_{1}\left(p_{x_{1}}\right)\right\rangle$ is the normal state (Landauer) conductance and $G_{11}(0)=\frac{4 \mathcal{N}_{1}}{R_{q}}\left\langle\frac{\left|v_{x_{1}}\right|}{v_{F}} D_{1}^{2}\left(p_{x_{1}}\right)\right\rangle$ is the subgap (BTK) conductance of the first NS interface.

Turning now to the non-local resistance

$$
R_{12}(T)=\frac{G_{12}(T)}{G_{11}(T) G_{22}(T)-G_{12}(T) G_{21}(T)},
$$

we substitute the expressions (10) and (9) into Eq. (12) and, neglecting the small cross-conductance term $G_{12} G_{21}$ in the denominator, we obtain

$$
\begin{aligned}
R_{12}(T)= & \frac{2 R_{N_{12}} e^{-\Delta / T}}{\left[G_{11}(0) / G_{N_{11}}+\sqrt{2 \pi \Delta / T} e^{-\Delta / T}\right]} \times \\
& \times \frac{1}{\left[G_{22}(0) / G_{N_{22}}+\sqrt{2 \pi \Delta / T} e^{-\Delta / T}\right]},
\end{aligned}
$$




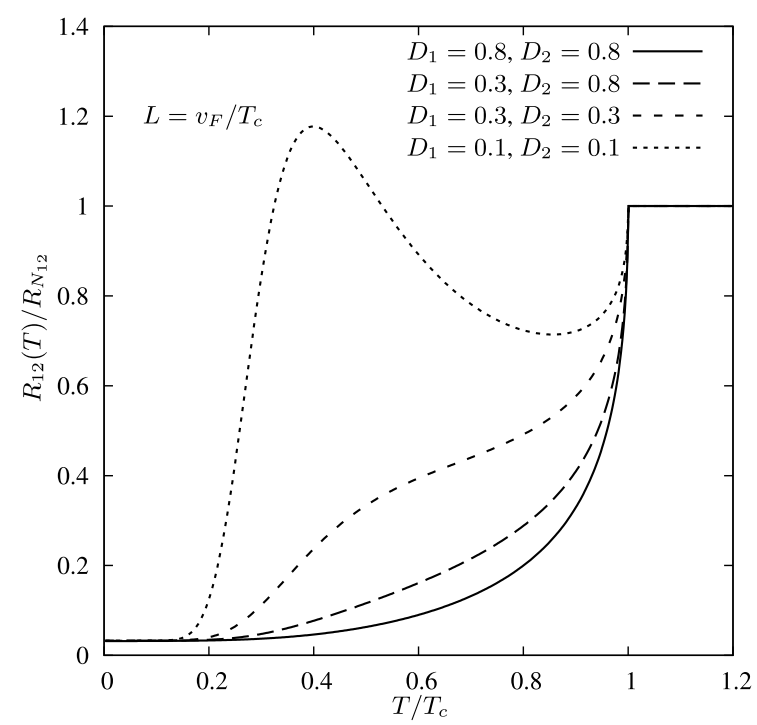

FIG. 2: Non-local resistance $R_{12}$ of an NSN device as a function of temperature for $L=v_{F} / T_{c}$. Provided both interface transmissions are sufficiently low the non-local resistance exhibits a well pronounced peak. This peak disappears at higher barrier transmissions.

where $R_{N_{12}}=G_{N_{12}} /\left(G_{N_{11}} G_{N_{22}}\right)$ is the cross-resistance in the normal state. Eq. (13) applies within the interval $D_{1} D_{2} \ll e^{-\Delta / T} \ll 1$ and represents the central result of this paper.

We observe that the non-local resistance (13) first increases with decreasing $T$ reaching its maximum at $T=T^{*}$ and then decreases with $T$ at lower temperatures. For the temperature $T^{*}$ with the logarithmic accuracy we obtain

$$
T^{*} \simeq \frac{\Delta}{\ln \sqrt{\frac{G_{N_{11}} G_{N_{22}}}{G_{11}(0) G_{22}(0)}}},
$$

or simply $T^{*} \simeq \Delta / \ln (1 / D)$ for symmetric structures with $D_{1,2}=D$. This result matches qualitatively with that obtained in Ref. ${ }^{11}$ within a different model.

The temperature dependence of the non-local resistance $R_{12}(T)$ for our NSN device is depicted in Fig. 2 for different values of the interface transmissions. In the limit of low transmissions the resistance $R_{12}(T)$ decreases with $T$ right below the superconducting critical temperature $T_{c}$ but then turns upwards and exhibits a well pronounced peak. At lower $T$ the resistance $R_{12}(T)$ decreases sharply and eventually tends to $R_{12}(0) \equiv$ $G_{12}(0) /\left[G_{11}(0) G_{22}(0)\right]$ in the limit $T \rightarrow 0$. With increasing interface transmissions $D_{1,2}$ the peak gets less pronounced and eventually disappears, in which case the non-local resistance $R_{12}(T)$ decreases monotonously with temperature. The same feature can also be observed in Eq. (13).

As it is clear from Fig. 3 both the height and the form of the non-local resistance peak scale with the distance $L$ exactly as the normal state value $R_{N_{12}}$. For instance,

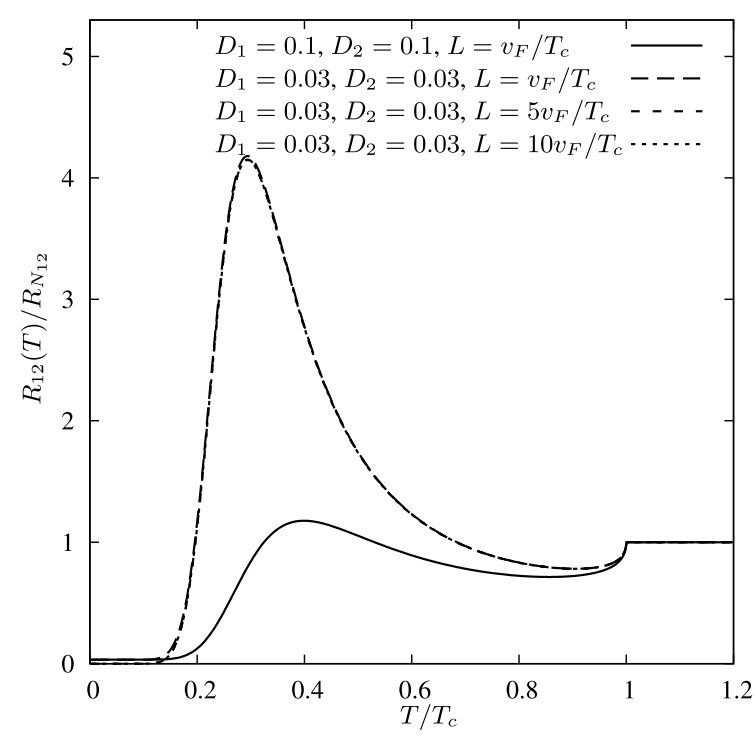

FIG. 3: The same as in Fig. 2 for different values of the distance $L$ between two N-terminals (measured in units of $\xi$ ). Three curves corresponding to the same barrier transmissions but different values of $L$ practically coincide, i.e. in the vicinity of the peak $R_{12}(T)$ in the superconducting state scales with $L$ exactly as $R_{N_{12}}$.

from Eq. (13) we easily determine the height of the resistance peak $R_{12}\left(T^{*}\right)$ which reads

$$
\frac{R_{12}\left(T^{*}\right)}{R_{N_{12}}}=\frac{\sqrt{\frac{2 T^{*}}{\pi \Delta}}}{\left[\sqrt{\frac{G_{11}(0)}{G_{N_{11}}}}+\sqrt{\frac{G_{22}(0)}{G_{N_{22}}}}\right]^{2}}
$$

We observe that the right-hand side of Eq. (15) is expressed only in terms of local conductances and, hence, is independent of $L$. This is an important result which might account for experimental observations 3 of a much weaker $L$-dependence of $R_{12}\left(T^{*}\right)$ as compared to the zero temperature value $R_{12}(0)$. For instance, within our model of ballistic electrodes for $L \gg \xi_{0}$ we have $R_{12}(0) \propto$ $\exp \left(-2 L \Delta(0) / v_{F}\right)$ while $R_{12}\left(T^{*}\right) \propto 1 / L^{2}$. Even weaker length dependence of $R_{12}\left(T^{*}\right) \propto R_{N_{12}} \propto 1 / L$ is expected in the diffusive limit. It would be interesting to scale the data $^{3}$ for the resistance peak at different lengths $L$ with the corresponding normal state resistance $R_{N_{12}}(L)$.

Although our theory correctly accounts for some key features of the experimental data it is important to bear in mind that the model employed here deals with ballistic electrodes connected via metallic constrictions whereas in experiments 1,3 the electrodes were most likely diffusive. In addition, we disregarded any relaxation mechanisms for non-equilibrium quasiparticles inside the superconductor (except for their escape into the normal terminals) while such mechanisms (caused, e.g., by electron-phonon and electron-electron interactions) are obviously present in experiments being responsible for a finite charge im- 
balance length. Hence, one can also expect certain differences. For instance, within our model the non-local resistance peak occurs only at small interface transmissions while the authors 1.3 observed such a peak at moderately high transmissions of NS interfaces. This might be an indication to relatively more pronounced charge imbalance effects.

In summary, we have developed a quantitative theory describing the peak in the temperature dependence of the non-local resistance recently observed in threeterminal NSN devices $\frac{1,3}{2}$ at sufficiently high $T$. This peak emerges as a result of a trade-off between quasi- particle/charge imbalance and subgap (Andreev) contributions to local and non-local conductances of the device. Both the height and the shape of the peak scale with the normal state resistance $R_{N_{12}}$ thus demonstrating much weaker (power law) dependence on the superconductor thickness $L$ as compared to the zero-temperature resistance $R_{12}(0)$ which decays (roughly) exponentially with increasing $L$.

We acknowledge stimulating discussions with D. Beckmann, V. Chandrasekhar and especially with D.S. Golubev. This work was supported in part by RFBR grant 06-02-17459.
1 D. Beckmann, H.B. Weber, and H. v. Löhneysen, Phys. Rev. Lett. 93, 197003 (2004); D. Beckmann and H. v. Löhneysen, Appl. Phys. A 89, 603 (2007).

2 S. Russo, M. Kroug, T.M. Klapwijk, and A.F. Morpurgo, Phys. Rev. Lett. 95, 027002 (2005).

3 P. Cadden-Zimansky and V. Chandrasekhar, Phys. Rev. Lett. 97, 237003 (2006).

${ }^{4}$ G. Falci, D. Feinberg, and F.W.J. Hekking, Europhys. Lett. 54, 255 (2001).

${ }^{5}$ R. Melin and D. Feinberg, Phys. Rev. B 70, 174509 (2004); R. Melin, ibid. 73, 174512 (2006).

6 A. Brinkman and A.A. Golubov, Phys. Rev. B 74, 214512 (2006).

7 J.P. Morten, A. Brataas, and W. Belzig, Phys. Rev. B 74, 214510 (2006).

8 M.S. Kalenkov and A.D. Zaikin, Phys. Rev. B 75, 172503 (2007).
${ }^{9}$ A. Levy Yeyati, F.S. Bergeret, A. Martin-Rodero, and T.M. Klapwijk, Nat. Phys. 3, 455 (2007).

10 S. Duhot and R. Melin, Phys. Rev. B 75, 184531 (2007).

11 D.S. Golubev and A.D. Zaikin, Phys. Rev. B 76, 184510 (2007).

12 M.S. Kalenkov and A.D. Zaikin, Phys. Rev. B 76, 224506 (2007); Physica E 40, 147 (2007).

13 For a review see, e.g., W. Belzig, F.K. Wilhelm, C. Bruder, G. Schön, and A.D. Zaikin, Superlatt. Microstruct. 25, 1251 (1999).

14 A.V. Zaitsev, Sov. Phys. JETP 59, 1015 (1984).

15 A.V. Galaktionov and A.D. Zaikin, Phys. Rev. B 65, 184507 (2002).

16 G.E. Blonder, M. Tinkham, and T.M. Klapwijk, Phys. Rev. B 25, 4515 (1982). 